\title{
Intelligent Evaluation System for Cross-culture Communications Competence
}

\author{
Xiaojuan Peng \\ Yunnan Technology and Business University, Kunming, China, 651701, China.
}

\begin{abstract}
Nowadays cross culture-communications is a primary objective in order to build a medium for communications. Although, it can be reviewed that due to different inhabitants most of the personalities faced impacts upon the communications, while the person is travelling or transferred to another region for official work. With regard to this impact, here in this paper, an intelligent evaluations system is being proposed that basically work upon the pursuing the language information between two separate inhabitants. Here, in this paper, a design of intelligent system through the applications of Android and artificial intelligence is being discussed that helps in developing a system that may reduce the impacts upon the communities.
\end{abstract}

Keywords: Cross-culture communications, intelligent evaluations system, android app, Google translate.

\section{Introduction}

Recently a large number of peoples are being travelled from one country to other, in terms of tourism, company's suggestions and lead a new lifestyle. The primary effects that they used to face within the main context of these effectivities are comprised as cross-culture communications gaps. The major impacts reviewed while the person could not be able to communicate with a single universal communications language. Particular to this impacts the placement of the embassies is being provided were the persons could find the relief in accordance with their satisfaction for fulfilling their demands. Moreover, with the development of the technical aspects, a new number of innovative applications are also present in the digital market that helps in providing communications among the personalities of varied in culture. Basically, an enhances order to effects are being seen while the companies used to transfer their employees from one country to another in accordance to attain the same collaborations of talents contributions to different manufacturing plants.

Here in this report, a different built-in intelligent evaluation system is being theoretically established that helps a varied number of persons in order to accompany the proper communication with different cultural inhabitants. Therefore, the satisfaction upon the development of communication can be accompanied for the tourists, which will provide safer associations in accompanied countries. In the below segment, the detailed descriptions of the intelligent evaluations systems upon the applications of android apps is being demonstrated briefly. The major constituents comprise of designing an android application that works upon the process of conducting the crosscultural communications among the users. Along with its justification in real-world application is also provided in the below segments. Therefore, the reader could easily identify the achievements that can be contributed regarding the practical implementation of the systems in real-world.

\section{The concept of Cross Culture Communications Competence}

The term cross culture communions have a varied number of descriptions. Basically, it occurs while the disturbance upon the communication is being greater in terms of not being able to understand certain language while the transmitted language is being contributed with the single description of language [1]. For example, while a person from China first invades in Australia. The major difference upon communicates language would lead impacts upon the personal communications. That further led to the disturbances upon the receiver person. Due to this particular reason, the difference or disturbance upon the communications is being innovated that cannot help in accompanies the communications transactions [2]. 
In effect to, his communications distractions a varied number of language conveying dictionaries are already being contributed into a number of countries. That while the users get to another inhabitant's area, he/she will not face any sorts of disturbances upon the communications process. For better instance, a large number of digitals apps are also present within the digital market that helps in seeking and gentrification a new skill upon the particular languages conveying in the real world. Like the Spanish learning apps, French learning apps are being provided within the digital market so that the users would interact with another native person of another country more efficiently. This is being established in order to reduce the impacts upon the travellers in order to perceive a communications process of another inhabitant. A varied number of communication process are already being established in the research world, basically, the transcript of Google plays a vital role in accompanying the supportive contributions upon the transactions of language form one inhabitant to another cultural inhabitant. But the basic difference within this type of communications that the persons are being able to communities through the written process of communication, rather than being able to pronounce the words of the varied community's perceptions. Further it can be demonstrated that a speaker pronunciation is also available within the communication process that ensures the contributions of refuge persons within the inhabitant area. But the major drawback to these methods is its long-time consuming for generations the knowledge of pronouncing different language.

In order to reduce this drawback a large number of institutes are also involved that helps in developing the skill within a single or limited time period. But in real field applications, the other contribution disturbances are also being accompanied with the seeker, such as the lack of interest in gathering the information upon gathering the basics of the other language [3]. Furthermore, the difference in cultural communication is also being seen while the global companies are transactions their employees from a place of the globe to another in order to accompany the talent to the number of manufacturing plants of the whole organization. In addition to these contents the companies used to locate a different housing complex for the personality, but the primary effects are accompanied with the communication with the co-workers and local inhabitants are being communicated for the person.

\section{The concept of Intelligent Evaluation System}

The terminology intelligent evaluations systems are comprised with traditional evaluation upon the development of the high-tech productions in order to provide the satisfaction of the lifesaving constituencies. This is demonstrated that the latest technical aspects lead to the development of the latest technological attributions upon the development of the identified gaps. The high-technical products involvement does not just help in enhancing the lifestyle of the persons but also contributes the most efficient contributions upon their daily working process [4]. Basically, within developing countries, the daily perceptions of the lifestyles of every individual are comprised of the implementation of the latest technological products. For example, regarding fast transportation peoples used to travels with the aeroplanes, that is constituents under the high-tech applications. Then for fast transporting from lower ground level to the upper level of the certain building, accelerations are being attributed that helps in processing the fast transportations. Basically, it can be demonstrated that from the hair cutter appliances to the Neil current products are all comprises under the productions of the high-tech applications [5].

The enhanced usage of the technical aspects is better contributed from the time when the companies have introduced the digital products within the market. The foremost popular product is the computer that helps in storing a greater number of data and also contributing the easily in working process for a number of employees within the organisation. Recently it has turned out to be the vital contributions for the peoples in order to do their works in a more easy and convenient way. Then the applications of the different search engines, cloud storing facilities and internet of things applications help in enhancing the applications of the technical aspects of the human's usage in today's applications. For example, Google is providing the emailing facility along with a greater number of applications 
for its users. Along with it the extra cloud facility it is associating for the users in order to store a number of documents along with confidentiality files. This contribution of Google along with the assistantships helps the users in a greater number of ways. Devoutly upon the contributions on the companies and organisations assumptions, Google has also implemented a lot of productive contributions that help the users to profound more attributed working aspects [6]. The better enhance of the high-technical aspects are being contributed by a large number of MNCs of all around the globe, that helps a number of users in order to satisfy their certain demands on real-field applications.

Introduction of these technical aspects is basically generated for fulfilling the gaps of the working strategies as well as helping the working effectively for the users. More often to build these sorts of products for the users, the compilers are also incremented the ways of handling the field of programming platforms. For example, initially, the $\mathrm{C}$ programming language is big introduced within the market that holds high-level contributions upon the building up certain products in practical applications. Then come to the java application that also helps in advancing the products more elaborative in nature, this turning the world of programming worlds, as it provides more efficient collaboration upon the basic C programming strategies. Now, the latest development of programming fields such as python, and radar applications proves more beneficial contributions upon the processing of the field applications.

\section{Design of an Intelligent System for Communications}

Here in this segment, the basilica discussion is contributed upon the development of certain intelligent evolution system that helps in reducing the cross-cultural communication that is being occurred in huge rate in today world. In order to develop this system, the chosen applications are identified as an android application that further helps in attracting a greater number of users worldwide. As mobile turns out to be the best provinces in today's market that helps a number of users to convey the required essentiality through the process of communication or storing their memories. Due to this particular reason, a theory is being contributed in order to develop an android app that has enhanced contributions within other operating systems, such as the windows, Linux and so on. In order to develop this application, we prefer the usage of the android applications as it constitutes more effective efficiency upon implementation [7].

For developing the applications, the most essential requirements is android suitable platform eclipse. That operates under the coding of Java applets. The layout interface that is being involved within the implications is designed in such a way that it conveys with the supportive contributions with the wireless IEEE 802.11a. That means one user have to talk in one end of a mobile phone and the other user can receive the single or transcript message within another mobile, where both of the devices are being connected through a WIFI connection. In order to develop the operations of the working strategy the interface of strings from the Google translate. That helps in contributing the transactions of words exchange from one language to another for the effective constituencies of the users. Another decision-making algorithm is also being implemented within the single application that helps in contributing the most accurate words attachment with the implications. Initially, the measurement of faulty assumptions is contributed experimentally through the interface of Linux. That helps in to identify the percentage of faulty contributions of words accompanied during the communications process. It is found to be around $30 \%$ faulty transaction is being achieved within the whole transactions of around 10-minute conversations. In order to reduce this faulty contribution to the attachment of the artificial intelligence upon the contribution of the communication process is also being attained. Due to not profound much effective contribution in real field applications, as it only reduces the faulty contributions but not allocating the perfect word contribution upon the process of communications.

In order to dissolve this issue, the major contributions of the neural networking systems upon the application processing provides most effective contributions regarding the accuracy of selecting words. This is also being analysed that the implementation of this particular networking facility is very much expensive upon the contributions for the reel's applications. Due to this particular reason 
an extra cloud facility is being allocated in order to perform the operations of the networking process through the cloud proficiencies. But implementation of this within the devices involves the increment of time consumptions for the conversations of the communication process. In order to resist the consumptions of time during operations, the imperative of processors percentage of contributions is being increased. During this process, it has been found that the pressure upon the processor is increased to such a high rate that it further causes and effects upon the mobile applications.

Due to this particular reason, the implementation of the conversation's technology is being prescribed with the original implementation processing factors. It can be determined that the major drawback of this applications is basically comprised the software implications, that is being initiated through the real applications of the internet connections. This helps in the efficient transaction of the communications data to the other inhabitant languages as the user-prescribed.

\section{Justifications of the Intelligent Evolutions System}

For implementing the real field implications of the most profound contributions of the essence is not being conveyed well enough due to certain factors. So, the accurate justification is not being contributed within this part of the implications. But still, the measuring software proficiencies are being contributed that helps in assuming the rate of processing of the overall assumption of the working strategies. For better transactions for the communication process, an extra hardware implementation is most essentially identified that helps in the processing of the overall workings' strategies. But still, the occupied contributions of the working policies help in attributing the most essential implementations of the gaps identified. That app is almost comprised of $75 \mathrm{MB}$ of data that is due to the provided inferences and daily compiling procedures of the operations management of the whole applications. At the same time, as the rough test upon the real field applications to a traveller's mobile, the obtained results are basically contributed upon the accuracy of pronunciation and at the same time, a time-consuming process is also being initialized within the overall process. This helps in identifying the gaps that need to be implemented within the application was that the better performance of the application can be achieved through the process of operations.

But the overall process of the applications is assumed to the acted more effectively rather than another number of communications devices present within the market. For better checking the application, a test of the application with a locally made product is being teased that helps in determining the certain number of assumed criteria that are needed to be implemented. Upon the justification of the overall process to come to be assumed that the overall process of the applications helps in determining the communications process. For better efficiency, a chatting process is also implemented within the algorithms that help in determining the communications process through a written strategy, which also enables the youngsters to contribute a greater number of words within the dictionary of the application [8]. The storing facility is being allocated within the devices so that the better practical implementation to the whole process is assumed to be the real-life applications of the Android apps.

Due to this particular reason number of attractions regarding the operation of the implications needs to be identified within the overview of the communications. For better statistical application an implementation of extra GPS system is also contributed within the applications that are being operated with Google Maps guidance. That helps the users to identify the persons of the other persons that are having the similar applications regarding pursuing the communications process. This also ensures that the process of the communication between the transmitter and the receiver persons can be conducted in an effective way. And also reduces the conflictive contributions regarding the processing of the operations of the communications from the number of users who are using it. It can be better assumed that the overall applications of these devices are being to most attractive contributions to number or tourist's personnel who used to roam from one end to another end. This ensures that the perfect or accuracy of the communications process for them to the contributions while the implementation of this software is being prohibited by the number of users. The major drawbacks 
for these sorts of users are being identified that the charging capacity of the devices is contributed most attractive contributions regarding the processing of the overall operations [9].

Upon justifications, it can be determined that the accuracy of the software is attracting a number of consumers demands, but the consumption of electricity is such increased in huge that e that it puts effects upon the processing of the operations that the application is being prescribed to do in real action. This can also be identified that in order to reduce the processing of operating upon the consumption of the overall power of the devices the better contributions of the hardware slots and another strategic algorithm is needed to be implemented within another hardware devices that can be attached with the personal body. So that why they conveyed way information trophy one end of the speakers then the traction of data or communication language will be changed for the received personnel.

\section{Conclusion}

In the concluding portion, it can be stated that the intelligent evaluation system is an indispensable portion of every individual's life. Communication is one of the most important aspects of any individual life and it can be stated that cross culture communication does indeed exist even today in the modern world. Such gaps can lead to problems and rise of issues which would otherwise not take place. The researcher with the help of the presented project has tried the best to bridge such a communication gap. The researcher has planned and implemented a theoretically based design which can be used in the presented situation or can be used to bridge the communication gaps between foreigners who inhabit two different cultures. Thus, it can be said that the communication gap does exist but it surely doesn't mean that an individual will have to live with it. With the help of theoretical frameworks and particular workings along with its proper implementation, such problems of communicative nature can be solved in a cost-efficient and effective manner. Thus, concluding this particular project, it may be said that cross-cultural communication will not pose to be a big problem in the future world of globalisation and advanced forms and means of communication.

\section{Future Work}

The future implementation of the whole application with the devices structures helps the users to accompany more efficient communicative processes. A better body attachment of these devices helps in contributions more effective contributions regarding communication with different language inhabitants. These contributions of other hardware contribution of these software products are also enhancing the production of high-tech applications within the market applications. A separate decision making and health assurance safety sensors implementation would help the more attractions for this particular product within the market. It can be assumed that the software implementations for the devices will help in reduction of the cross-cultural communications affectivity, and at the same time also attracts a number of users around the globe.

\section{References}

[1]. Milovanova, A. V., and O. V. Salikaeva. "Cross-cultural communication." Проблемы геологии и освоения недр: труды XXI Международного симпозиума имени академика МА Усова студентов и молодых ученых, посвященного 130-летию со дня рождения профессора МИ Кучина, Томск, 3-7 апреля 2017 г. Т. 2.-Томск, 2017.2 (2017): 1017-1018.

[2]. Weber, Elena A. "Heritage Interpretation as Tool in Cross-Cultural Communication: Challenges and Solutions in the Work of Guides-Interpreters." (2018).

[3]. Koopialipoor, Mohammadreza, Danial Jahed Armaghani, Ahmadreza Hedayat, Aminaton Marto, and Behrouz Gordan. "Applying various hybrid intelligent systems to evaluate and predict slope stability under static and dynamic conditions." Soft Computing (2018): 1-17. 
[4]. Hu, S. Y., E. Reel, and A. Scheer. "Challenges in cross-cultural communication in breast cancer surgery: Is there a gender gap." European Journal of Cancer 92 (2018): S84.

[5]. Seera, Manjeevan, and Chee Peng Lim. "A hybrid intelligent system for medical data classification." Expert Systems with Applications 41, no. 5 (2014): 2239-2249.

[6]. Duffy, Melissa C., and Roger Azevedo. "Motivation matters: Interactions between achievement goals and agent scaffolding for self-regulated learning within an intelligent tutoring system." Computers in Human Behaviour 52 (2015): 338-348.

[7]. Wu, Hong. "Research on the Cross-Cultural Communication of TV Dance Show: Taking So You Think You Can Dance as the Example." DEStech Transactions on Social Science, Education and Human Science assume (2016).

[8]. Yin, Shen, and Xiangping Zhu. "Intelligent particle filter and its application to fault detection of a nonlinear system." IEEE Transactions on Industrial Electronics 62, no. 6 (2015): 3852-3861.

[9]. Adler, Nancy J., and John L. Graham. "Cross-cultural Interaction: The International Comparison Fallacy." In Language in International Business, pp. 33-58. Palgrave Macmillan, Cham, 2017. 